\title{
Effects of Antenatal Uteroplacental Hypoperfusion on Neonatal Microvascularisation and Excitotoxin Sensitivity in Mice
}

\author{
JULIE CATTEAU, JAINE-ISCIA GERNET, STÉPHANE MARRET, HÉLÈNE LEGROS, PIERRE GRESSENS, PHILIPPE LEROUX, \\ AND VINCENT LAUDENBACH
}

\begin{abstract}
EA 4309 "NeoVasc" [J.C., J.-I.G., S.M., H.L., P.L., V.L.], Normandy-Rouen University Institute for Biomedical Research, IFRMP23,
76183 Rouen, France; Department of Neonatal Pediatrics and Intensive Care [S.M., V.L.], Rouen University Hospital Charles Nicolle, 76031 Rouen, France; INSERM, U676, PremUP [P.G.], Faculté de Médecine Denis Diderot, Université Paris 7, 75019 Paris, France
\end{abstract}

\begin{abstract}
Vascular intrauterine growth restriction (IUGR) occurs in about $5 \%$ of pregnancies and may reduce the incidence of periventricular leukomalacia in preterm newborns. We evaluated neonatal excitotoxicity in a murine model of vascular IUGR involving unilateral uterine ligation on embryonic day (E)13.5. Birth weight was significantly decreased in the ligation group compared with the sham group ( $p<0.001)$. VEGFs, VEGF receptors (VEGFRs), and NMDA receptor subunit mRNAs in brain extracts were assayed using quantitative RT-PCR. Ligation was associated with increased mRNAs for the vascular marker PECAM-1 on postnatal day (PD)2 and VEGFR-3 on PD2 and PD10, contrasting with decreased VEGFA and VEGFC on PD10. Microvessel density was increased on PD7. Ligated and sham pups received intracerebral ibotenate (NMDA agonist) on PD2 or PD10. Cortical and white matter (WM) lesions after $5 \mathrm{~d}$ were reduced in ligated versus sham pups injected on PD2 ( $p<0.001$ and $p<0.01$, respectively); this effect persisted on PD42 ( $p<0.01$ and $p<0.05$, respectively). With ibotenate on PD10, lesions were exacerbated after $5 \mathrm{~d}$ in the ligated group in the cortex $(p<0.05)$ and WM $(p<0.05)$ and on PD42 in the cortex $(p<0.05)$. In conclusion, vascular IUGR offered only transient protection against neonatal excitotoxic lesions, possibly via angiogenesis. (Pediatr Res 70: 229-235, 2011)
\end{abstract}

$\mathrm{I}^{\mathrm{n}}$ ntrauterine growth restriction (IUGR) complicates up to 5\% of pregnancies (1). Complications of IUGR include fetal distress requiring preterm delivery and neonatal neurological abnormalities such as periventricular leukomalacia (PVL) $(2,3)$. A large body of evidence supports a pivotal role for the excitatory neurotransmitter glutamate in the pathophysiology of perinatal brain lesions (4). Glutamate induces overactivation of ionotropic receptors [i.e. $N$-methyl-D-aspartate (NMDA) and $\alpha$-amino-3-hydroxy-5-methylisoxazole-4propionate, AMPA-kainate] and metabotropic receptors, thereby triggering intracellular events known as the excitotoxic cascade, which ultimately causes neural cell death $(4,5)$. Most cases of IUGR are due to vascular disorders associated with abnormal placental growth and blood flow (1). Data on

Received November 29, 2010; accepted February 28, 2011.

Correspondence: Philippe Leroux, Ph.D., EA4309 NeoVasc, Normandy-Rouen University Institute for Biomedical Research, School of Medicine-Pharmacy, 22, Bd Gambetta, F-76183 Rouen Cédex, France; e-mail: philippe.leroux@univ-rouen.fr

Supported by University of Rouen, the Institut National de la Santé et de la Recherche Médicale (INSERM), the Société Française d'Anesthésie Réanimation (SFAR), ELA Foundation, the European Found for Regional Development (FEDER), the Regional Council of Haute-Normandie, and the National Research Agency (ANR).

J.C. and J.I.G. contributed equally to this work. the relationship between vascular IUGR and brain lesions in newborns are conflicting. Some studies suggested a reduced risk of PVL in preterm newborns with vascular IUGR compared with other preterm infants $(6,7)$. In contrast, more recent cohort studies found no decrease in neurological disabilities in premature newborns with vascular IUGR associated with maternal hypertension (8). Thus, whether impaired placental blood flow affects the neonatal brain remains unclear.

Vascular IUGR can be viewed as a model of antenatal ischemic preconditioning (9-11). The term "preconditioning" designates numerous approaches used to elicit endogenous mechanisms, usually aimed at affording protection from further insults. Preconditioning may involve exposing organs (usually the heart or brain) to a broad range of conditions such as hypoxia-ischemia (9), lipopolysaccharides $(12,13)$, cobalt (14), or volatile anesthetics (15). In a previous study, we showed that postnatal hypoxic preconditioning exerted protective effects in a murine model of excitotoxic brain lesions induced by intracerebral (i.c.) injections of the NMDA glutamate agonist ibotenate (16). This neuroprotective effect involves the vascular endothelial growth factor (VEGF)/VEGF receptor (VEGFR)-2/Flk-1 pathway, which has neurotrophic and angiogenic properties (16). Few experimental studies focused on the potential for antenatal ischemic preconditioning to exert neuroprotective effects in the event of a neonatal insult. Olivier et al. studied rat pups with vascular IUGR induced by unilateral uterine artery ligation on embryonic day (E) 17.5 and exposure to a cerebral excitotoxic insult on postnatal day 5 (PD5). White matter (WM) lesion size was smaller in pups with moderate growth restriction and larger in pups with severe growth restriction, compared with control animals $(11,17)$. The mechanisms involved in this differential effect of antenatal ischemia remain partly unclear. More specifically, the potential role for the time of ischemic preconditioning and/or age at postnatal excitotoxic insult remains to be determined.

\footnotetext{
Abbreviations: AUVL, antenatal uterine vessel ligation; E, embryonic day; i.c., intracerebral; NMDA-R, glutamate receptors of the $N$-methyl-D-aspartic acid type; PD, postnatal day; PECAM-1, platelet endothelial cell adhesion molecule-1, CD-31; PVL, periventricular leukomalacia; VEGFR, VEGF receptors (types 1, 2 or 3); WM, white matter
} 
We hypothesized that IUGR caused by reduced uterine blood flow in pregnant mice may have preconditioning like effects on the brain of the offspring subjected to injury at different postnatal ages. The aim of this study was to investigate the effects of antenatal uterine artery ligation on neonatal expression of VEGFs and their receptors, brain vascularization, NMDA receptor expression, and sensitivity to neonatal NMDA-induced neurotoxicity.

\section{METHODS}

Animals. Animal care and housing were in accordance with guidelines issued by the Institut National de la Santé et de la Recherche Médicale (INSERM). Protocols were approved by the Normandy Regional Ethics Committee for animal experimentation, in accordance with French legislation (authorizations N/01-07-07/08/08-10 and N/04-09-06/17). Experiments were carried out on female Naval Medical Research Institute mice and their pups (Janvier, Le Genest Saint Isle, France). Animals were kept under normothermic conditions with a $12 \mathrm{~h} / 12 \mathrm{~h}$ light/dark cycle and water and chow ad libitum.

Uterine artery ligation protocol in pregnant mice. The surgical procedure was performed on E13.5. Anesthesia was induced by isoflurane (Mundipharma, Issy-les-Moulineaux, France). Body temperature was maintained using a hotplate throughout surgery. Double permanent ligation of the left uterine horn containing the left uterine artery and vein was performed using surgical wire (Vicryl 4.0; Ethicon, Auneau, France). The sham procedure consisted in a laparotomy and exposure of the uterus outside the abdominal cavity without ligation. The number of embryos was counted in the ligation and control groups. After surgery, the wound was infiltrated with bupivacaine (Astra-Zeneca, Rueil-Malmaison, France; $2.5 \mathrm{mg} / \mathrm{kg}$ ). All animals were maintained on the hotplate until they regained consciousness. Live pups were counted at birth. Fetal losses were measured as the differences between live births and in utero counts of fetuses at the time of surgery, expressed as percent. Each experimental group included at least two pregnant mice and their litters. All live pups from any female included were considered for the study without consideration of body weight.

Quantitative RT-PCR for VEGFs, VEGFRs, platelet endothelial cell adhesion molecule-1, and NMDA-R subunits. The telencephalons of newborns from two litters in each group were collected on PD2 $(n=19-24)$ or PD10 $(n=22-26)$, immediately frozen, and stored at $-80^{\circ} \mathrm{C}$ until use. Total RNA was extracted using a NucleoSpin RNA II kit (Macherey-Nagel, Düren, Germany), and the RNA concentration was determined based on the OD at $260 \mathrm{~nm}$. Then, $1 \mu \mathrm{g}$ of RNA was reverse transcribed (Promega, Charbonnières, France).

Primers (Proligo, Paris, France; Table 1) were designed from GenBank mouse sequences using Primer Express V 1.0 software (Applied Biosystems). Quantitative RT-PCR was carried out in an ABI 7500 real-time PCR system using SYBR Green Mastermix (Applied Biosystems, Courtaboeuf, France) cDNA was amplified for 40 cycles, and expression of each gene was normalized for expression of glyceraldehyde 3-phosphate dehydrogenase mRNA.

Evaluation of brain vascular density in newborn mice. The effect of antenatal uterine vessel ligation (AUVL) on cortical vascular density was evaluated by immunolabeling of platelet endothelial cell adhesion molecule- 1 (PECAM-1) or CD-31, a specific marker for endothelial cells. Pups were anesthetized with isoflurane (Mundipharma) on PD2, PD7, PD10, or PD15 and given an intracardiac perfusion of paraformaldehyde (4\% diluted in $0.1 \mathrm{M}$ PBS, pH 7.4; Sigma Chemical Co.). Brains were postfixed in $4 \%$ paraformaldehyde in PBS for $48 \mathrm{~h}$. Forty micrometer slices were cut on a vibrant microtome (VT1000S; Leica Biosystems, Nussloch, Germany). The sections were stained with rat anti-PECAM-1 antibody (1:100; MEC 13.3; BD Pharmingen, Le Pont-De-Claix, France). Revelation was with an avidin-biotin horseradish peroxidase kit (Vector Laboratories, Burligame). At least five randomly selected animals from two different litters were included in each group, and immunoreaction was performed for six sections per brain. Vascular density in the frontoparietal lobes was estimated from the number of labeled vessel segments (length $\geq 10 \mu \mathrm{m}$ ) per square millimeter determined by an investigator who was unaware of group assignment and who used stereological analysis software Mercator (Explora-Nova, LA Rochelle, France).

Intracerebral injection of excitotoxic drugs in newborn mice. Excitotoxic lesions of the developing brain were induced using stereotactic i.c. injections of the NMDA glutamate receptor agonist ibotenate (Sigma Chemical Co., St.-Quentin, France; $10 \mu \mathrm{g} / 2 \mu \mathrm{L}$ in sterile PBS per animal) as previously described $(16,18-21)$. Injections were performed on PD2 or PD10 under isoflurane anesthesia using a 26-gauge needle mounted on a calibrated Hamilton microdispenser, inserted $2 \mathrm{~mm}$ (on PD2) or $3 \mathrm{~mm}$ (on PD10) below the external surface of the scalp in the frontoparietal area of the right hemisphere. Two $1-\mu \mathrm{L}$ boluses were injected $10 \mathrm{~s}$ apart, and the needle was left in place for 10 additional seconds. Under these conditions, excitotoxins target both the cortex and the underlying periventricular intermediate zone on PD2, which has become WM by PD10. The pups were returned to their dams immediately after the injections.

Brain lesion size assessment in newborn mice. Lesion size was determined $5 \mathrm{~d}$ after ibotenate injection or in adulthood (PD42), as previously described $(18-21)$. The pups were killed by decapitation at different times after lesion induction (PD7, PD10, or PD42), depending on the experiment. The brains were removed, fixed in $4 \%$ formaldehyde for $7 \mathrm{~d}$, and embedded in paraffin. Former studies showed a strong correlation between the maximal radial and frontooccipital diameters of the ibotenate-induced lesions $(18,21)$. Consequently, we cut serial $10-\mu \mathrm{m}$ sections from the frontal to the occipital pole in the coronal plane. All sections were stained with cresyl violet and examined by an investigator who was unaware of group assignment. Maximal lesion size was computed as the number of sagittal sections containing a visible lesion multiplied by $10 \mu \mathrm{m}$.

Statistical analysis. The data were described as mean \pm SD (Q1-Q3). Results were compared using the following tests: fetal loss, Fisher's exact test; birth weight and vascular density, unpaired $t$ test; brain lesion size, two-way ANOVA followed by unpaired $t$ test; mRNA levels were measured by quantitative RT-PCR, one-way ANOVA followed by Bonferroni posttest. All statistical analyses were performed using Prism version 4 software (Graphpad Software, San Diego, CA).

\section{RESULTS}

Effect of AUVL on in utero fetal loss and birth weight. The mean number of embryos was $14 \pm 3$ in both the sham and the ligation group. At birth, litter size was $12 \pm 3$ and

Table 1. Sequences of primers used for quantitative PCR

\begin{tabular}{|c|c|c|}
\hline Genes & Forward & Reverse \\
\hline GAPDH & 5'-CATGGCCTTCCGTGTTCCTA-3' & 5'-CCTGCTTCACCACCTTCTTGA-3' \\
\hline PECAM & 5'-TCCAACAGAGCCAGCAGTATGAG-3' & 5'-TCCAATGACAACCACCGCAATG-3' \\
\hline$V E G F A$ & 5'-CCCTGGCTTTACTGCTGTACCT-3' & 5'-TGGGACTTCTGCTCCTTCTG-3' \\
\hline$V E G F B$ & 5'-GGCAACACCAAGTCCGAATG-3' & 5'-GCTGTGTTCTTCCAGGGACATC-3' \\
\hline$V E G F C$ & 5'-CCTCAGCAAGACGTTGTTTGAA-3' & 5'-AGGAAGTGTGATTGGCAAAACTG-3' \\
\hline$V E G F D$ & 5'-CTGCAGCTGTGAGGACAGATG-3' & 5'-AAGCGCCAGTGCTTTCCA-3' \\
\hline VEGFR1 & 5'-GGGTCCTCGTTCCAGTCTTC-3' & 5'-CGGCTGCTTCCGATGTTT-3' \\
\hline VEGFR2 & 5'-GATGCAGGAAACTACACGGTCAT-3' & 5'-GGAATCCATAGGCGAGATCAAG-3' \\
\hline VEGFR3 & 5'-GGTTCCTGATGGGCAAAGG-3' & 5'-TCAGTGGGCTCAGCCATAGG-3' \\
\hline NR1 & 5'-CTCTAGCCAGGTCTACGCTATCC-3' & 5'-GACGGGATTCTGTAGAAGCCA-3' \\
\hline$N R 2 A$ & 5'-ACATCCACGTTCTTCCAGTTTGG-3' & 5'-GACATGCCAGTCATAGTCCTGC-3' \\
\hline$N R 2 B$ & 5'-CCAGAGTGAGATGGATTGC-3' & 5'-TGGGCTCAGGGATGAAACTGT-3' \\
\hline$N R 3 A$ & 5'-ATCCTCAAGCGCATCGGACA-3' & 5'-CGACTCTGGCTCATCCCTCTG-3' \\
\hline$N R 3 B$ & 5'-GAGCTTCCCCGAGATGCA-3' & 5'-CTATGGACACCTCATAATCCAGTAGTG-3' \\
\hline
\end{tabular}


$10 \pm 3$ in the sham and AUVL groups, respectively. Mean fetal loss was $14.4 \pm 12.9 \%$ and $26.8 \pm 22.0 \%$ in the sham and AUVL groups, respectively ( $p<0.001$, Fisher exact test). Birth weight was significantly lower in the AUVL group than in the sham group $[1.52 \pm 0.20(1.35-1.70)$ g versus $1.80 \pm$ $0.15(1.73-1.91)] \mathrm{g}$, respectively; $p<0.001)$. A weight difference persisted until PD10 (not shown).

Effect of $A U V L$ on vessel density in the newborn brain. Typical patterns after PECAM-1 immunolabeling are shown in Figure $1 A$ and $B$. AUVL increased the vascular density in the neonatal cortex compared with sham litters on PD7 $\left(940.3 \pm 88.2\right.$ versus $703.4 \pm 88.5$ vessels $/ \mathrm{mm}^{2}, p<0.001$; Fig. $1 D$ ) but not on PD2, PD10, or PD15 (Fig. $1 C, E$, and $F$ ). PECAM-1 immunolabeling of the WM was too faint to be measured.

Effect of AUVL on VEGFs, VEGFRs, PECAM-1, and NMDA receptor subunit $m R N A s$ in the newborn brain. In newborn brains collected between PD2 and PD10 in the sham group, declines occurred in mRNAs for VEGFB $(p<0.05)$, VEGFC $(p<0.01)$, VEGFD $(p<0.001)$, and VEGFR-3 $(p<$ 0.01; Fig. 2). AUVL did not modify the expression on PD2 of

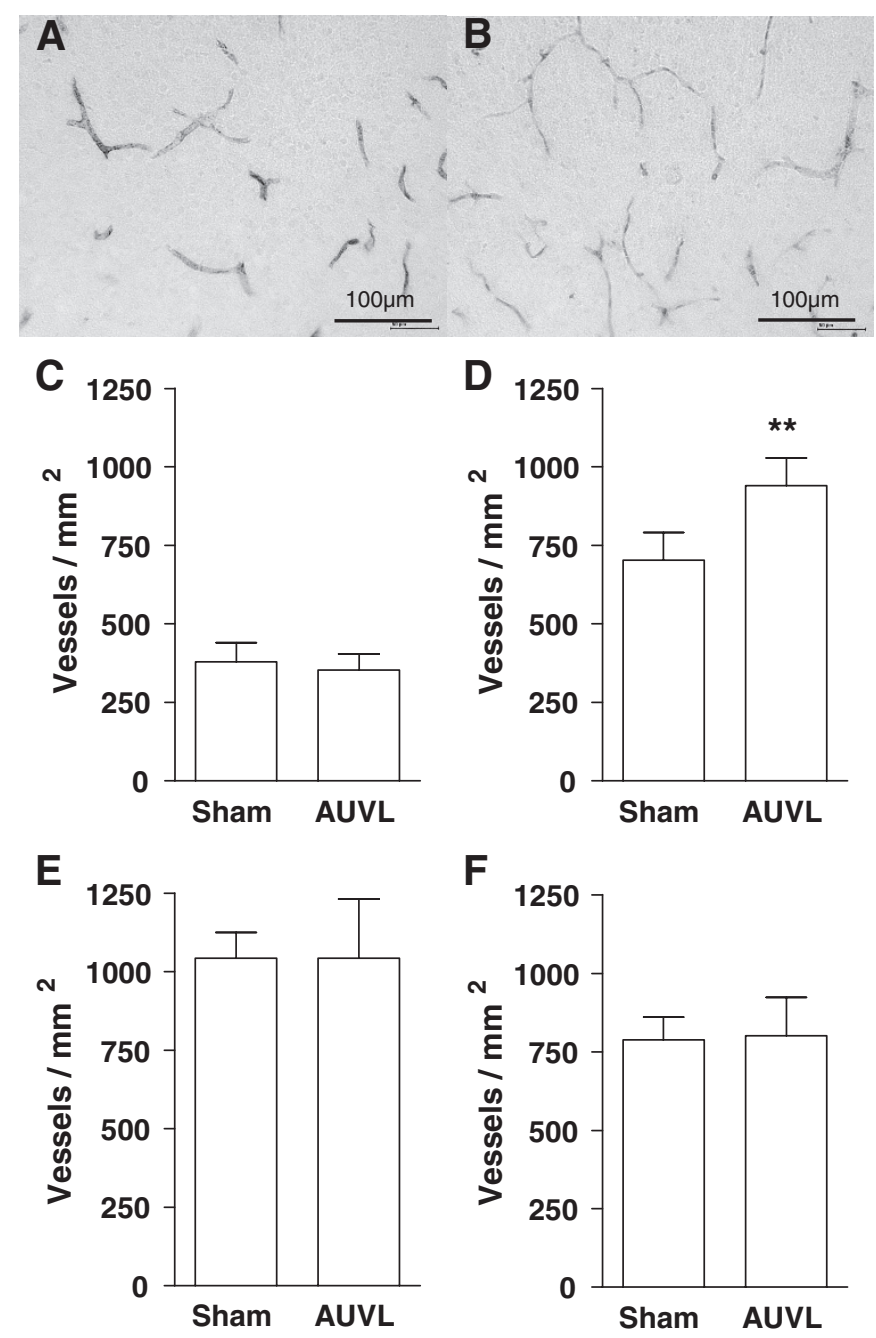

Figure 1. Immunostaining for PECAM-1. Typical aspects seen in the brains of animals on PD7 after a sham operation $(A)$ or AUVL $(B)$. Scale bar $=100$ $\mu \mathrm{m}$. Brain vascular density on PD2 $(C), \operatorname{PD} 7(D), \operatorname{PD} 10(E)$, or PD15 $(F)$. $*^{*} p<0.001$ vs sham. Bars: vessels per $\mathrm{mm}^{2} \pm \mathrm{SD}(n=6)$.
VEGFA, VEGFB, VEGFC, VEGFD, VEGFR-1, or VEGFR-2. Conversely, VEGFR-3 mRNAs and PECAM-1 mRNAs were significantly increased $(p<0.05)$ on PD2 (Fig. 2 ). AUVL brains studied on PD10 exhibited decreased expression of VEGFA and VEGFC and increased expression of VEGFR3 $(p<0.05)$. No difference in PECAM-1 mRNA was found between the sham and AUVL groups (Fig. 2).

In sham animals, mRNAs for the NMDA receptor subunits NR1 and NR2A increased between P2 and P10 $(p<0.001)$, whereas NR2B and NR3B mRNAs decreased $(p<0.05$ and $p<0.001$, respectively; Fig. 3). After AUVL, the only change in expression levels of these mRNAs on PD2 or PD10 was decreased NR3B mRNA on PD2 ( $p<0.05$; Fig. 3).

Effect of AUVL on excitotoxic brain lesion size in newborn mice. In sham newborns, ibotenate induced WM atrophy and/or cavitary lesions surrounded by focal cortical necrosis (Fig. 4). Ibotenate on PD2 after AUVL on E13.5 induced smaller cortical lesions compared with sham animals, on both PD7 and PD42 (Fig. 4B). The cortex lesion size decrease in the AUVL group was highly significant $[p<0.0001$ by two-way ANOVA; $413 \pm 187 \mu \mathrm{m}$ versus $817 \pm 220 \mu \mathrm{m}$ on PD7 $(p<0.001)$ and $277 \pm 216 \mu \mathrm{m}$ versus $510 \pm 208 \mu \mathrm{m}$ $(p<0.01)$ on PD42; Fig. 5A]. AUVL also diminished the WM lesions $[p<0.001$ by two-way ANOVA; $200 \pm 148 \mu \mathrm{m}$ versus $354 \pm 159 \mu \mathrm{m}$ on PD7 $(p<0.01)$ and $212 \pm 199 \mu \mathrm{m}$ versus $390 \pm 233 \mu \mathrm{m}(p<0.05)$ on PD42; Fig. 5B]. Conversely, after ibotenate injection on PD10, AUVL was associated with significantly larger cortical lesions $[p<0.001$ by two-way ANOVA; $750 \pm 235 \mu \mathrm{m}$ versus $604 \pm 212 \mu \mathrm{m}(p<$ $0.05)$ on PD15 and $717 \pm 296 \mu \mathrm{m}$ versus $494 \pm 175 \mu \mathrm{m}(p<$ 0.05) on PD42; Fig. 5C]. Regarding WM lesions, two-way ANOVA revealed an interaction between the effect of ligation and age at observation $(p<0.01)$. AUVL increased WM lesion size on PD15 (435 $\pm 193 \mu \mathrm{m}$ versus $185 \pm 146 \mu \mathrm{m}$, $p<0.05)$ but had no effect on PD42 (435 $\pm 221 \mu \mathrm{m}$ versus $444 \pm 268 \mu \mathrm{m}$; Fig. 5D).

\section{DISCUSSION}

We found that effects of experimental AUVL in pregnant mice included neonatal growth restriction, increased brain microvascular density, alterations in VEGFs and VEGFRs expression 2 and $10 \mathrm{~d}$ after birth, and alterations in NMDAR-induced excitotoxicity during early and late postnatal development, with virtually no change in NMDA-R expression.

The model used consists in ligation of the left uterine horn, which blocks the flow of blood through the uterine artery and vein. The uterine vasculature is characterized by numerous anastomoses, which supply blood to the ligated side, explaining the very low mortality rate in our study. About half the fetuses are exposed to blood flow restriction. Labeling these fetuses in utero is not feasible. We therefore studied the full litters. Theoretically, the presence of animals from the nonligated uterine horn in the AUVL groups might result in heterogeneous changes in markers affected by blood flow restriction. However, Q3 of birth weights in AUVL pups was below Q1 in sham group, indicating that growth restriction in AUVL pups likely affected not only the fetuses located in the 
A
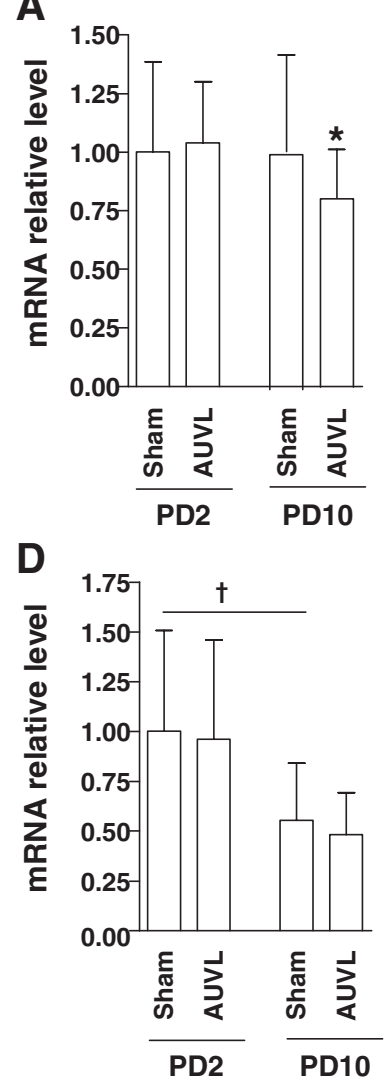

B

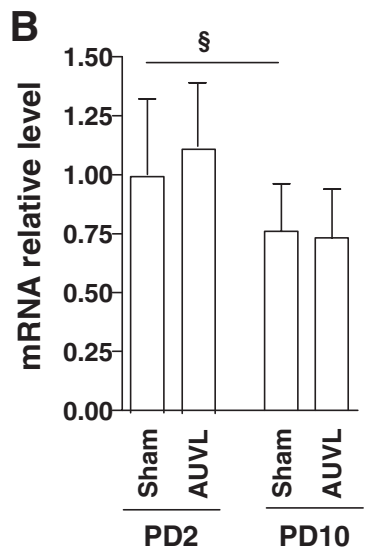

E

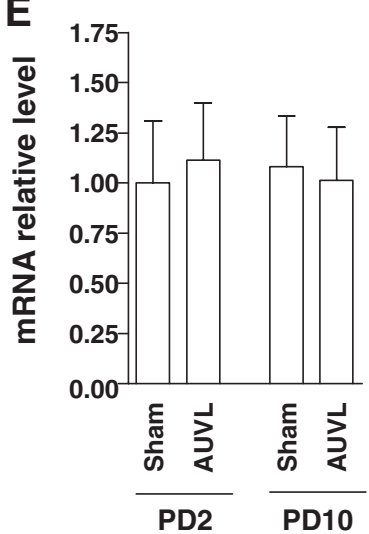

C

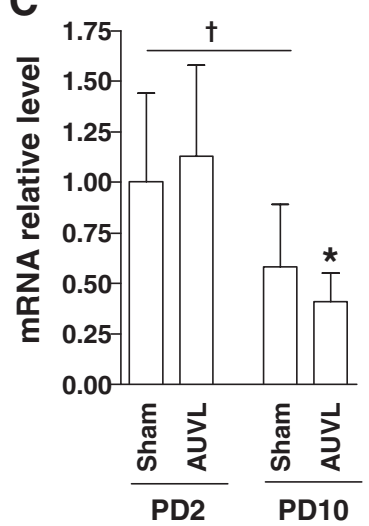

$\mathbf{F}$

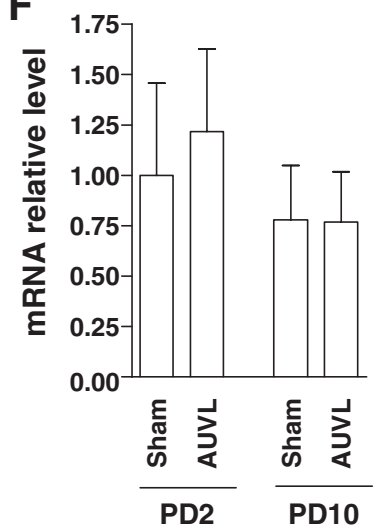

Figure 2. Vascular gene expression profile (quantitative RT-PCR). Relative levels of VEGFA $(A)$, VEGFB $(B)$, VEGFC $(C)$, VEGFD $(D)$, VEGFR-1 $(E)$, VEGFR-2 $(F)$, VEGFR-3 $(G)$, and PECAM-1 $(H)$ mRNA (normalized for glyceraldehyde 3-phosphate dehydrogenase mRNA expression) in the cortex on PD2 (sham, $n=19$; AUVL, $n=24$ ) and PD10 (sham, $n=26$; AUVL, $n=22$ ). $* p<0.5 v s$ sham; $\S p<0.05$ vs PD2; $\dagger p<0.01$ vs PD2. Bars: normalized mRNA \pm SD.
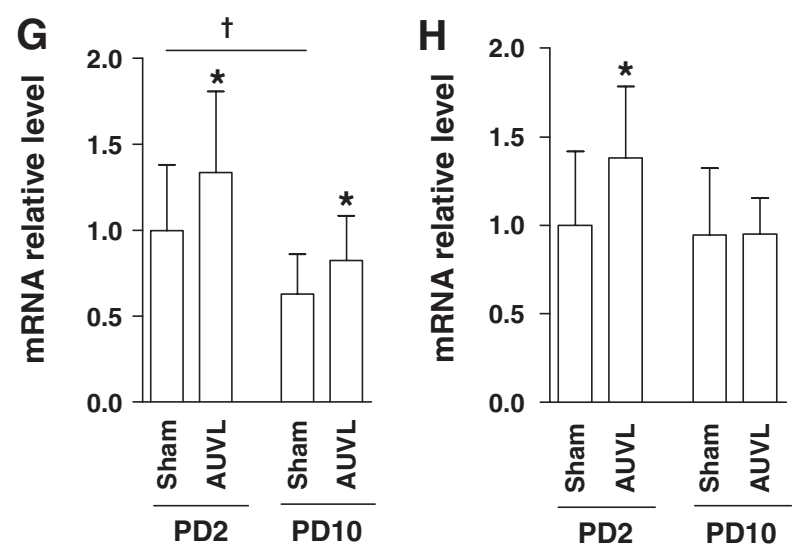

ligated uterine horn, and that the whole litter may be considered homogeneously.

The expression of several gene products belonging to the VEGF family (VEGFB, VEGFC, VEGFD, VEGFR2, and VEGFR3) decreased between PD2 and PD10 in the sham animals. The only significant effect of AUVL on these products was a significant increase in VEGFR3, a receptor specifically involved in angiogenesis during development and whose activity is restricted to lymphangiogenesis in adult (22). This increase may explain the enhanced PECAM expression in the ligation group on PD2, the increased microvessel density on PD7, and the reduced lesion size observed at PD7 in AUVL pups injected with ibotenate at PD2. Conversely, the enhanced VEGFR3 expression on PD10 was not associated with increases in PECAM expression or vascular density on PD10 and PD15, perhaps as a result of decreased expression of VEGFA and of VEGFC, the preferential ligand for VEGFR3 (22). These modest changes in VEGFs and their receptors several days after in utero blood flow restriction differ markedly from findings in ischemic areas of postmortem human neonatal brains, where increases in VEGFA and VEGF-R2 are the most prominent features (23). Therefore, our findings in mice with IUGR probably reflect an alteration in the schedule of vascular ontogenesis, with early angiogenesis on PD7 and normalization as early as PD10.

AUVL significantly decreased ibotenate-induced cortical and WM lesions on PD2, by nearly 50\%, whereas it slightly but significantly worsened lesions induced on PD10. Quantification of mRNAs for NMDA receptor subunits showed no significant alterations in the expression of these receptors. Thus, changes in these receptors cannot explain the increased NMDA agonist toxicity, in contrast for instance to data from 
A

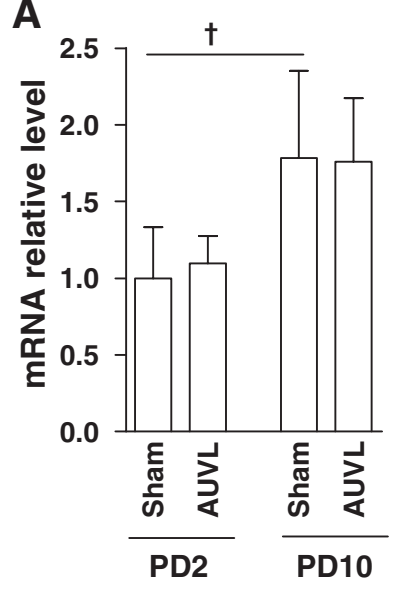

D

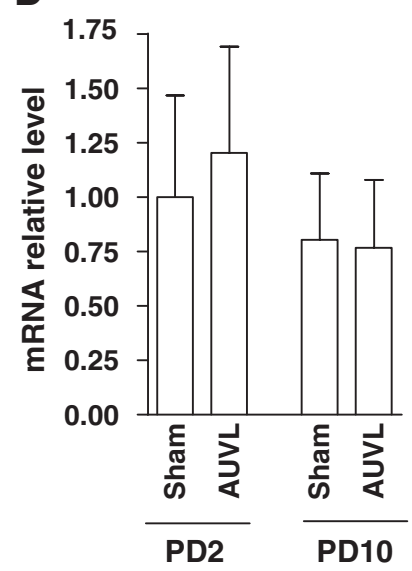

B

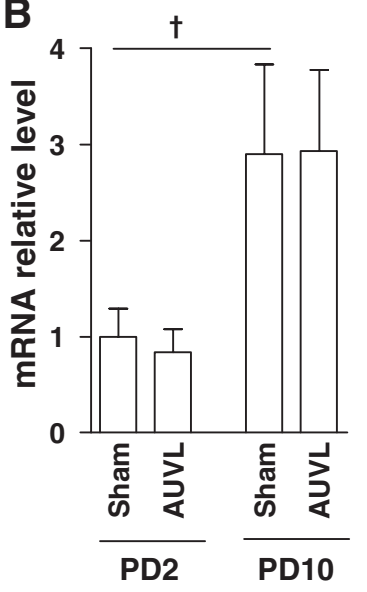

C

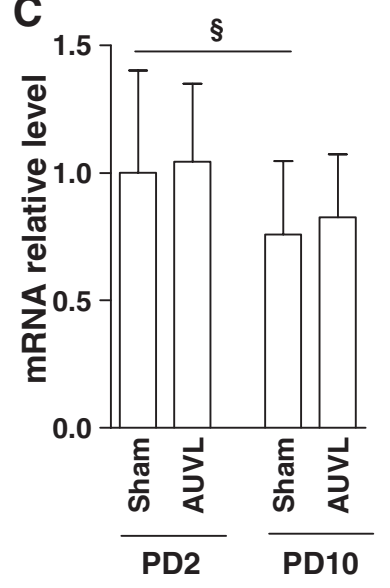

Figure 3. NMDA receptor subunit expression profile (quantitative RT-PCR). Relative levels of NMDA receptor subunit NR1 $(A)$, NR2A $(B)$, NR2B $(C)$, NR3A $(D)$, and NR3B $(E)$ mRNA (normalized for glyceraldehyde 3-phosphate dehydrogenase mRNA expression) in the cortex on PD2 (sham, $n=19$; AUVL, $n=24$ ) and PD10 (sham, $n=26$; AUVL, $n=22$ ). ${ }^{*} p<0.5 v s$ sham; $\S p<0.05$ vs PD2; $\dagger p<0.001$ vs PD2. Bars: normalized mRNA \pm SD.

\section{E}

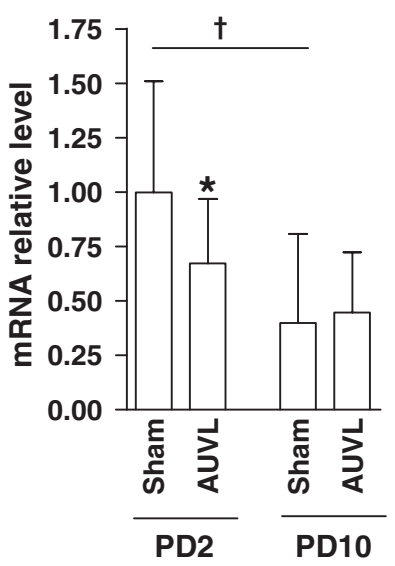

A

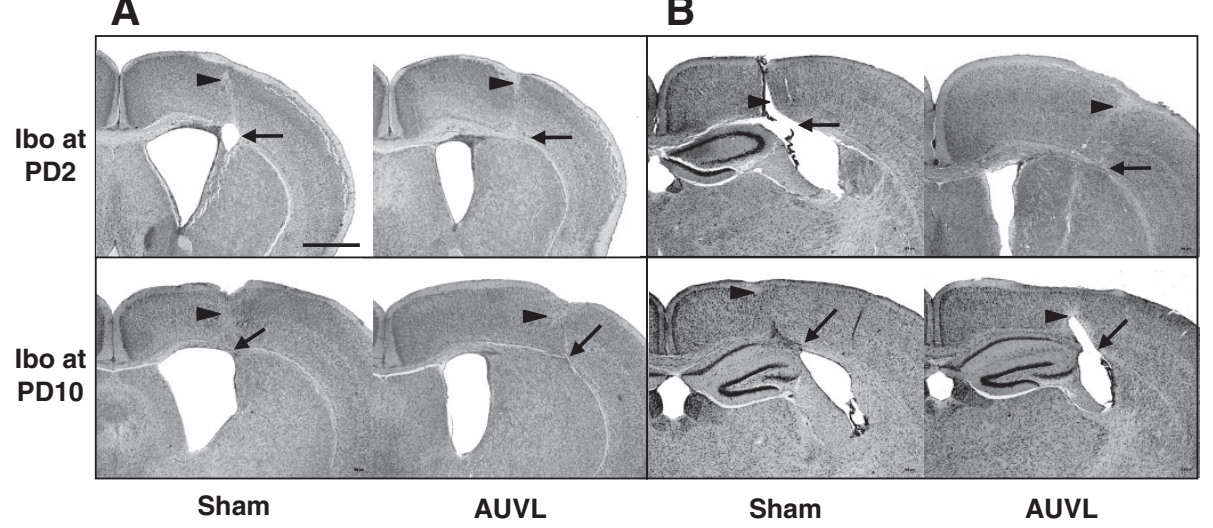

Figure 4. Typical features of cresyl violetstained brain sections from mice given $2 \mu \mathrm{g}$ of ibotenate (Ibo) as an i.c. injection on PD2 or PD10. (A) Brains $5 \mathrm{~d}$ after i.c. injection; $(B)$ adult brains on PD42. Arrows, WM lesions (atrophy, cyst). Arrowheads, cortical necrosis. Scale bar: $800 \mu \mathrm{m}$. neonates born to dams subjected to chronic mild stress during pregnancy (24). This finding suggests that AUVL may alter NMDA receptor function downstream from receptor expression. The timing of the effect of AUVL on ibotenate toxicity, with a protective effect initially and a deleterious effect later on, suggests a transient preconditioning mechanism $(16,17)$. Previous work suggests that VEGFs and their receptors may play pivotal roles in various preconditioning paradigms $(9,16,25,26)$. However, the mechanisms involved in the effects of AUVL seemed different from VEGFR2-mediated neuroprotection after postnatal hypoxia (16). Age is a critical parameter that must be taken into account when evaluating the influence of a single injury. Our data from sham-operated animals confirm our earlier finding (27) that ibotenate-induced lesions are smaller when produced on PD10 than when produced on PD2, in the cortex and, to a lesser extent, the WM. This result does not constitute evidence for involvement in the protective effect of VEGFs and their receptors, whose mRNA expression declined between P2 and P10 in sham pups. Preconditioning strategies have different consequences depending on the age of the animal at the time of preconditioning and/or injury $(12,17)$. Preconditioning induced by antenatal exposure to a low dose of lipopolysaccharide sensitizes the neonatal brain to hypoxia-ischemia but decreases the severity of brain 

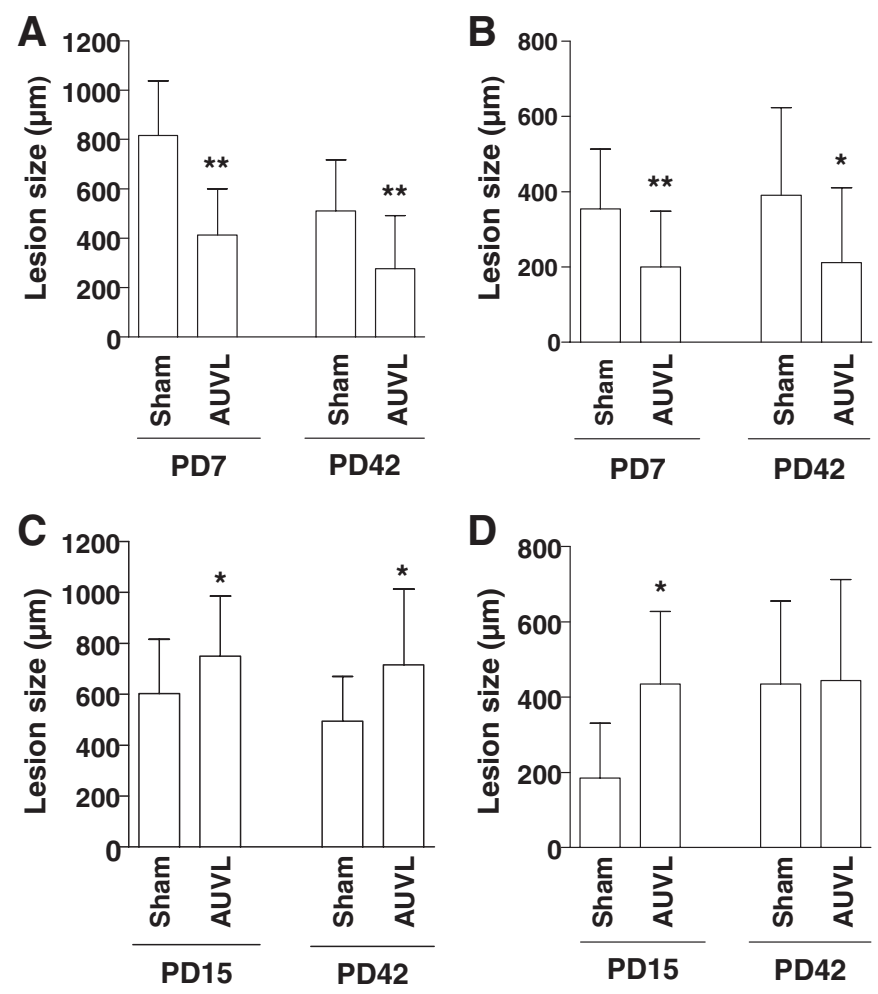

Figure 5. Lesion size after ibotenate injection in pups born after AUVL or a sham operation. Mean size of cortical and WM lesions induced by i.c. ibotenate (Ibo) injected on PD2 and examined at PD7 (sham, $n=14$; AUVL, $n=18$ ) or PD42 (sham; $n=10$; AUVL, $n=26$ ) or injected at PD10 and examined at PD15 (sham, $n=27$; AUVL, $n=20$ ) or PD42 (sham, $n=17$; AUVL, $n=18$ ). (A) Cortical lesions after Ibo injection on PD2; (B) WM lesions after Ibo injection on PD2; $(C)$ cortical lesions after Ibo injection on PD10; $(D)$ WM lesions after Ibo injection on PD10. $* p<0.05, * * p<0.01$ between effects of Ibo in AUVL vs sham-operated litters. Bars: mean lesion size \pm SD.

injury in adult animals (13). Our finding of differential effects of a single insult depending on the time since AUVL indicates that, after the putative preconditioning effect affording neuroprotection on the PD2-PD7 period, lasting changes occurred in the brain of pups exposed to AUVL sensitizing them to excitotoxicity of ibotenate. These effects might be concomitant but masked at earlier terms due to anticipated vascularization that would be protective. Irrespective of the effects found $5 \mathrm{~d}$ after ibotenate injection, the decrease or amplification of its toxicity in AUVL pups observed early after the ibotenate injection was still present in adulthood, ruling out long-term AUVL effects on recovery.

Numerous studies in women with preeclampsia or fetuses having IUGR showed alterations in VEGFs, PIGF, and their receptors in the placenta, and increases in soluble VEGFR1 (sFlt-1) in the peripheral bloodstream (28-30). However, these markers failed to predict the outcome. Conversely, retrospective studies showed that restricted uterine blood flow, preeclampsia, or IUGR were associated with decreased rates of PVL $(7,8)$ or CP $(6)$ in preterm infants. Our model of i.c. ibotenate injection on $\mathrm{P} 2$ or P10 has been validated as accurately replicating excitotoxic lesions in human preterm or full-term neonates $(21,27)$. Our findings regarding excitotoxic lesion severity when ibotenate is injected early after AUVL
(PD2) are in line with the decreased incidence of short-term excitotoxic lesions in infants with IUGR. The enhanced sensitivity to excitotoxin on PD10 in the AUVL group is also consistent with the high rates of cognitive deficiency, behavioral problems, and school difficulties in older children born with IUGR (31). In theory, these disabilities may result from the environment later in infancy or from underlying neurodevelopmental defects masked by short-term protective effects of operant preconditioning in the early postnatal period. It would be of interest to perform cognitive tests in noninjured AUVL-exposed adult animals.

In conclusion, AUVL has differential effects depending on age at injury, and these effects are consistent with observations in human infants. Although the apparently neuroprotective effect of IUGR in the event of insults in early infancy may be viewed as beneficial, IUGR has detrimental long-term effects. Early neovascularization as a component of putative operant preconditioning may account for the transient neuroprotective effect. The mechanisms underlying lasting sensitization to injuries remain obscure. The AUVL model may help to elucidate the effects of IUGR and their underlying mechanisms, in particular, via studies of behavior and cognition in adult AUVL-exposed mice.

Acknowledgments. We thank Dr. S. Jegou-Colleter, Dr. B.J. Gonzalez, and Dr. P. Mulder for technical help and critical discussion.

\section{REFERENCES}

1. Mandruzzato G, Antsaklis A, Botet F, Chervenak FA, Figueras F, Grunebaum A, Puerto B, Skupski D, Stanojevic M 2008 Intrauterine restriction (IUGR). J Perinat Med 36:277-281

2. Goldenberg RL, Culhane JF, Iams JD, Romero R 2008 Epidemiology and causes of preterm birth. Lancet 371:75-84

3. Pallotto EK, Kilbride HW 2006 Perinatal outcome and later implications of intrauterine growth restriction. Clin Obstet Gynecol 49:257-269

4. Volpe JJ 2009 Brain injury in premature infants: a complex amalgam of destructive and developmental disturbances. Lancet Neurol 8:110-124

5. Olney JW 1993 Role of excitotoxins in developmental neuropathology. APMIS suppl40:103-112

6. Murphy DJ, Sellers S, MacKenzie IZ, Yudkin PL, Johnson AM 1995 Case-control study of antenatal and intrapartum risk factors for cerebral palsy in very preterm singleton babies. Lancet 346:1449-1454

7. Baud O, Zupan V, Lacaze-Masmonteil T, Audibert F, Shojaei T, Thebaud B, Ville Y, Frydman R, Dehan M 2000 The relationships between antenatal management, the cause of delivery and neonatal outcome in a large cohort of very preterm singleton infants. BJOG 107:877-884

8. Ancel PY, Marret S, Larroque B, Arnaud C, Zupan-Simunek V, Voyer M, Roze JC, Matis J, Burguet A, Ledesert B, Andre M, Pierrat V, Kaminski M 2005 Are maternal hypertension and small-for-gestational age risk factors for severe intraventricular hemorrhage and cystic periventricular leukomalacia? Results of the EPIPAGE cohort study. Am J Obstet Gynecol 193:178-184

9. Dirnagl U, Becker K, Meisel A 2009 Preconditioning and tolerance against cerebral ischaemia: from experimental strategies to clinical use. Lancet Neurol 8:398-412

10. Dirnagl U, Simon RP, Hallenbeck JM 2003 Ischemic tolerance and endogenous neuroprotection. Trends Neurosci 26:248-254

11. Olivier P, Baud O, Bouslama M, Evrard P, Gressens P, Verney C 2007 Moderate growth restriction: deleterious and protective effects on white matter damage. Neurobiol Dis 26:253-263

12. Wang X, Hagberg H, Nie C, Zhu C, Ikeda T, Mallard C 2007 Dual role of intrauterine immune challenge on neonatal and adult brain vulnerability to hypoxiaischemia. J Neuropathol Exp Neurol 66:552-561

13. Eklind S, Mallard C, Arvidsson P, Hagberg H 2005 Lipopolysaccharide induces both a primary and a secondary phase of sensitization in the developing rat brain. Pediatr Res 58:112-116

14. Xi L, Taher M, Yin C, Salloum F, Kukreja RC 2004 Cobalt chloride induces delayed cardiac preconditioning in mice through selective activation of HIF-1alpha and AP-1 and iNOS signaling. Am J Physiol Heart Circ Physiol 287:H2369-H2375

15. Sergeev P, da Silva R, Lucchinetti E, Zaugg K, Pasch T, Schaub MC, Zaugg M 2004 Trigger-dependent gene expression profiles in cardiac preconditioning: evidence for 
distinct genetic programs in ischemic and anesthetic preconditioning. Anesthesiology 100:474-488

16. Laudenbach V, Fontaine RH, Medja F, Carmeliet P, Hicklin DJ, Gallego J, Leroux P, Marret S, Gressens P 2007 Neonatal hypoxic preconditioning involves vascular endothelial growth factor. Neurobiol Dis 26:243-252

17. Olivier P, Baud O, Evrard P, Gressens P, Verney C 2005 Prenatal ischemia and white matter damage in rats. J Neuropathol Exp Neurol 64:998-1006

18. Husson I, Mesples B, Bac P, Vamecq J, Evrard P, Gressens P 2002 Melatoninergic neuroprotection of the murine periventricular white matter against neonatal excitotoxic challenge. Ann Neurol 51:82-92

19. Laudenbach V, Mantz J, Lagercrantz H, Desmonts JM, Evrard P, Gressens P 2002 Effects of alpha(2)-adrenoceptor agonists on perinatal excitotoxic brain injury: comparison of clonidine and dexmedetomidine. Anesthesiology 96:134-141

20. Gressens P, Marret S, Hill JM, Brenneman DE, Gozes I, Fridkin M, Evrard P 1997 Vasoactive intestinal peptide prevents excitotoxic cell death in the murine developing brain. J Clin Invest 100:390-397

21. Marret S, Mukendi R, Gadisseux JF, Gressens P, Evrard P 1995 Effect of ibotenate on brain development: an excitotoxic mouse model of microgyria and posthypoxiclike lesions. J Neuropathol Exp Neurol 54:358-370

22. Lohela M, Bry M, Tammela T, Alitalo K 2009 VEGFs and receptors involved in angiogenesis versus lymphangiogenesis. Curr Opin Cell Biol 21:154-165

23. Sentilhes L, Marret S, Leroux P, Gonzalez BJ, Laquerrière A 2011 Vascularendothelial growth factor and its high affinity receptor VEGFR-2 in the norma versus destructive lesions in human forebrain during development: an immunohistochemical comparative study. Brain Res 1385:77-86

24. Rangon CM, Fortes S, Lelievre V, Leroux P, Plaisant F, Joubert C, Lanfumey L, Cohen-Salmon C, Gressens P 2007 Chronic mild stress during gestation worsens neonatal brain lesions in mice. J Neurosci 27:7532-7540
25. Bernaudin M, Nedelec AS, Divoux D, MacKenzie ET, Petit E, Schumann-Bard P 2002 Normobaric hypoxia induces tolerance to focal permanent cerebral ischemia in association with an increased expression of hypoxia-inducible factor-1 and its target genes, erythropoietin and VEGF, in the adult mouse brain. J Cereb Blood Flow Metab 22:393-403

26. Bernaudin M, Tang Y, Reilly M, Petit E, Sharp FR 2002 Brain genomic response following hypoxia and re-oxygenation in the neonatal rat. Identification of genes that might contribute to hypoxia-induced ischemic tolerance. J Biol Chem 277:3972839738

27. Hennebert $\mathrm{O}$, Laudenbach V, Laquerrière A, Verney $\mathrm{C}$, Carmeliet $\mathrm{P}$, Marret $\mathrm{S}$, Leroux P 2005 Ontogenic study of the influence of tissue plasminogen activator (t-PA) in neonatal excitotoxic brain insult and the subsequent microglia/macrophage activation. Neuroscience 130:697-712

28. Tsatsaris V, Goffin F, Munaut C, Brichant JF, Pignon MR, Noel A, Schaaps JP, Cabrol D, Frankenne F, Foidart JM 2003 Overexpression of the soluble vascular endothelial growth factor receptor in preeclamptic patients: pathophysiological consequences. J Clin Endocrinol Metab 88:5555-5563

29. Savvidou MD, Yu CK, Harland LC, Hingorani AD, Nicolaides KH 2006 Maternal serum concentration of soluble fms-like tyrosine kinase 1 and vascular endothelial growth factor in women with abnormal uterine artery Doppler and in those with fetal growth restriction. Am J Obstet Gynecol 195:1668-1673

30. Boutsikou T, Malamitsi-Puchner A, Economou E, Boutsikou M, Puchner KP, Hassiakos D 2006 Soluble vascular endothelial growth factor receptor-1 in intrauterine growth restricted fetuses and neonates. Early Hum Dev 82:235-239

31. Leonard H, Nassar N, Bourke J, Blair E, Mulroy S, de Klerk N, Bower C 2008 Relation between intrauterine growth and subsequent intellectual disability in a ten-year population cohort of children in Western Australia. Am J Epidemiol 167:103-111 